\title{
Ultrashort electron bunch length measurement with diffraction radiation deflector
}

\author{
Dao Xiang* and Wen-Hui Huang \\ Department of Engineering Physics, Tsinghua University, Beijing, China, 100084
}

(Received 4 December 2006; published 4 January 2007)

\begin{abstract}
In this paper, we propose a novel method to measure electron bunch length with a diffraction radiation (DR) deflector which is composed of a DR radiator and three beam position monitors (BPMs). When an electron beam passes through a metallic aperture which is tilted by 45 degrees with respect to its trajectory, backward DR that propagates perpendicular to the beam's trajectory is generated which adds a transverse deflection to the beam as a result of momentum conservation. The deflection is found to be largely dependent on the bunch length and could be easily observed with a downstream BPM. Detailed investigations show that this method has wide applicability, high temporal resolution, and great simplicity.
\end{abstract}

DOI: 10.1103/PhysRevSTAB.10.012801

PACS numbers: 29.27.Fh, 41.60.- $\mathrm{m}, 41.75 . \mathrm{Ht}$

\section{INTRODUCTION}

There are growing interests in generation, measurement, and applications of short electron beam with energy ranging from a few $\mathrm{MeV}$ to hundreds of $\mathrm{GeV}$. The timeresolved ultrafast electron diffraction (UED) needs a few $\mathrm{MeV}$ femtosecond (fs) electron beam to visualize the fundamental microscopic phenomena [1-3]. The inverse Compton scattering facilities need a fs electron bunch to provide high flux and short x-ray pulse [4]. In the plasma wakefield acceleration experiment, an electron beam with temporal duration smaller than the plasma wavelength $(\sim 100 \mathrm{fs})$ is needed in order to generate a monochromatic beam [5]. The x-ray free electron lasers (XFEL) also utilize the short bunch together with high charge to provide a high peak current which initiates the instabilities that lead to efficient lasing in a single pass through the undulator [6]. Precise measurement of bunch length is necessary for developing such kinds of facilities. In addition to the high temporal resolution, the compatible method should also be able to preserve the beam qualities during the measurement, for all these facilities require a low emittance beam.

In the past decade, many methods have been developed to measure picosecond (ps) and fs electron bunch. A streak camera has been widely used to measure the temporal structure of the electron beam, but the resolution is limited to about $200 \mathrm{fs}$ even for the state-of-the-art technology [7]. A zero-phasing method has shown a few tens of fs temporal resolution [8], but it uses extra acceleration structures and is a destructive method. An electro-optic sampling method has recently called much attention and shown the ability of measuring sub-ps bunch [9-12], but the temporal resolution is limited to $\sim 100 \mathrm{fs}$ due to the transverse optical lattice oscillation in the electro-optic crystal [13]. Coherent radiation, e.g., coherent synchrotron radiation [14], transition radiation [15], and diffraction radiation [16,17] have

\footnotetext{
*Corresponding author.

Electronic address: xiangdao@tsinghua.org.cn
}

also been widely used in electron bunch length measurement, but they generally only measure the average bunch length with multishots and suffered from spectrum distortion problems.

Using coherent diffraction radiation (DR) to measure electron bunch length has been implemented in many laboratories, taking advantage of its nonintercepting characteristic. The method generally uses an interferometer or a spectrometer to obtain the radiation spectrum which is further used to predict the bunch length. The main problem of this method is that the accurate measurement of the spectrum is not trivial because the radiation measured by the detector could largely deviate from that originally generated by the electron bunch due to window transmission, diffraction loss, water and carbon oxide absorption in air, detector response, etc.

In this paper, we propose a new and nonintercepting method to measure electron bunch length with a DR deflector. The system is composed of a DR radiator which deflects the beam and three beam position monitors (BPMs) of which two are put upstream of the radiator to monitor the intrinsic trajectory variation and the third one downstream to measure the deflection caused by the DR radiator. The method is compatible to the facilities mentioned above, for the electron does not hit the radiator directly and the emittance growth due to Coulombic scattering as that suffered in the intercepting method is completely avoided. It is found the deflection is very sensitive to bunch length, which offers a simple method for bunch length determination. The weak dependence of the DR deflection on beam energy and charge indicates a great potential in applying this method to low charge low energy beam measurement. Furthermore, the shorter the bunch, the larger the deflection, which makes this method very suitable for short bunch measurement.

\section{PRINCIPLES OF DR DEFLECTOR}

DR is generated when there is optical inhomogeneity in space the presence of which would induce changing cur- 
rents that generate the radiation. Specifically the most widely studied case is that when DR is generated by relativistic electron beam passing through some aperture (rectangular slit, circular aperture, etc.) on a metallic target. When the target is infinitely stretched, the DR problem could be handled by solving Maxwell's equations [18]. But for practical conditions where the target always has limited transverse size, the properties of DR sometimes could largely deviate from that generated by infinite size target.

We have applied the diffraction model to effectively treat this problem [19]. In this model, the field of an electron is quantized into pseudophotons that are locked to the electron and cannot propagate freely. But when transmitted through or reflected by the metallic target, the pseudophotons can convert to real photons which propagate along the direction of velocity [forward DR (FDR)] and the specular reflection direction [backward DR (BDR)]. When the electron center passes through a circular disk with inner radius $a$ and outer radius $b$, the distribution for DR per solid angle per angular frequency can be written as [19]

$$
\begin{aligned}
\frac{d^{2} W}{d \omega d \Omega}= & \frac{e^{2}}{4 \pi^{3} \varepsilon_{0} c} \frac{\beta^{2}}{\gamma^{2}\left(1-\beta^{2} \cos ^{2} \theta\right)^{2}} \\
& \times\left[\Upsilon\left(\frac{\omega a}{2 \pi c \gamma}\right)-\Upsilon\left(\frac{\omega b}{2 \pi c \gamma}\right)\right]^{2}, \\
\Upsilon(x)= & 2 \pi x\left[J_{1}(2 \pi x \gamma \sin \theta) K_{2}(2 \pi x)\right. \\
& \left.-\gamma \sin \theta J_{2}(2 \pi x \gamma \sin \theta) K_{1}(2 \pi x)\right],
\end{aligned}
$$

where $\beta=v / c$ is the speed of the electron normalized with respect to that of light, $\gamma$ is the Lorentz factor, $J(x)$ and $K(x)$ are the Bessel function of the first kind and the modified Bessel function of the second kind, respectively, and $\theta$ is the polar angle measured from the beam's trajectory for FDR and the specular direction for BDR.

Consider the general case in beam diagnostics with DR where the target is tilted by 45 degrees with respect to the beam's trajectory. In this case, the propagation direction of BDR is perpendicular to the trajectory as shown in Fig. 1. As dictated by momentum conservation, the generation of BDR should result in a transverse deflection to the beam.

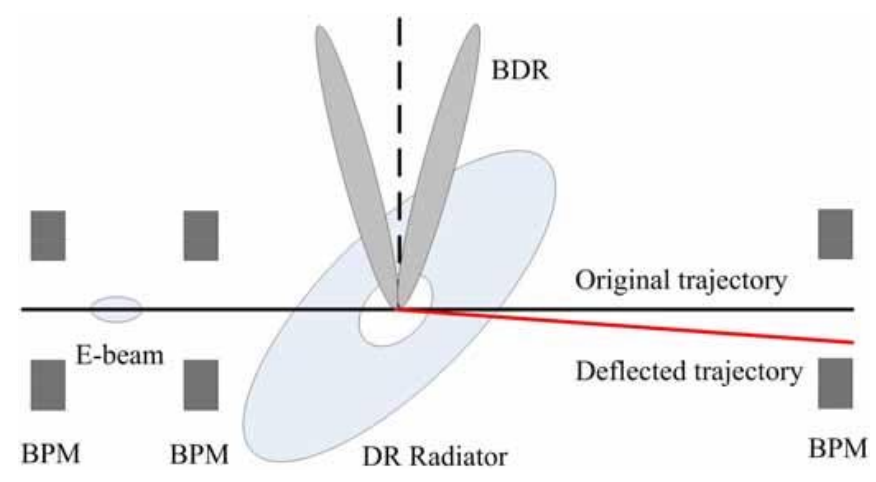

FIG. 1. (Color) The geometry of the DR deflector.
The deflection could also be understood as caused by the short-range wakefield. In our previous work we have performed a comparison study of the wakefield with transition radiation field [20] and diffraction radiation field [21]. It is demonstrated that they differ only in subjective terminology. The authors in [22] have shown the equivalence of describing the short-range wakefield with the radiation recoil effect.

For a single electron, the total energy taken by the BDR photons is found by integrating Eq. (1) in frequency and the whole space,

$$
I_{e}=\iint \frac{d^{2} I}{d \omega d \Omega} d \omega d \Omega
$$

The upper limit of the frequency in calculating the integration is generally taken to be the plasma frequency of the target material above which the surface currents concept and the pseudophoton diffraction model become invalid. One could easily find that the total energy taken by the BDR photons are negligibly small as compared to the energy of the electron. For instance, the total energy taken by the BDR photons is found to be $2 \times 10^{-5} \mathrm{eV}$ for an electron with energy $50 \mathrm{MeV}$ when it passes through an aluminum target with inner radius $3 \mathrm{~mm}$ and outer radius $30 \mathrm{~mm}$.

However, when electrons radiate collectively as that happened for an electron bunch, the radiation field whose wavelength is larger than the bunch length would add coherently and as a result the radiated energy per electron is increased by a factor that depends on bunch length and number of electrons within a bunch [23]. In this case, the energy loss for each individual electron is largely enhanced and the corresponding deflection is found to be

$$
r^{\prime}=\frac{\Delta p_{\perp}}{p}=\frac{1}{E} \iint \frac{d^{2} I \cos \theta}{d \omega d \Omega}[1+(N-1) F(\omega)] d \omega d \Omega,
$$

where $E$ is beam energy, $N$ is the electron number contained within one bunch, and $F(\omega)=\left|\int f(t) e^{i \omega t} d t\right|^{2}$ is the so-called longitudinal form factor which is just the square of the Fourier transform of the normalized longitudinal distribution $f(t)$. For a Gaussian beam with rms bunch length $\sigma_{z}$, the form factor is $F(\omega)=\exp \left(-\omega^{2} \sigma_{z}^{2} / c^{2}\right)$. The factor $\cos \theta$ in Eq. (2) takes into account the fact that the BDR propagates within a cone and only the perpendicular momentum affects the deflection while the parallel momentum cancels each other due to symmetry.

Based on Eq. (2), we propose a method to measure electron bunch length with a DR deflector. The deflection is converted to trajectory variation which can be measured with a BPM downstream. 


\section{APPLICATIONS IN BUNCH LENGTH MEASUREMENT}

We will estimate several cases with typical parameters to show the wide applicability of the DR deflector. For the accelerator based far infrared (FIR) source and inverse Compton scattering based x-ray source, the beam energy is typically a few tens of $\mathrm{MeV}$ and the required sub-ps bunch is provided either by magnetic bunch compressor (BC) or by velocity bunching. The XFEL typically utilize two-stage $\mathrm{BC}$ to provide very high peak current beam. The $\mathrm{BC}$ typically operates at a few hundred $\mathrm{MeV}$ and a few $\mathrm{GeV}$ respectively [6]. Consider a circular DR target with inner radius $3 \mathrm{~mm}$ and outer radius $30 \mathrm{~mm}$. The temporal structure of the beam is assumed to be Gaussian distribution and the beam charge is assumed to be $1 \mathrm{nC}$ which is generally used in designing such kinds of facilities. The deflection caused by DR target to the beam when the beam energy is $50 \mathrm{MeV}$ (typical value for most of the FIR and Compton scattering x-ray source) and $250 \mathrm{MeV}$ (typical value for the first BC of the XFEL) is calculated and shown in Fig. 2 as a function of bunch length.

If we assume the distance from the DR radiator to the $\mathrm{BPM}$ to be $1 \mathrm{~m}$, then $1 \mathrm{mrad}$ deflection would result in $1 \mathrm{~mm}$ variation of the trajectory. A BPM with resolution $<20 \mu \mathrm{m}$ is regularly available (a cavity BPM could have submicron resolution [24]), the trajectory variation could thus be easily observed.

One could see two general trends from Fig. 2. One is that the deflection is stronger for a beam with shorter bunch length. This characteristic may make this method very suitable for measurement of very short electron bunch. The other is that the deflection amplitude decreases as beam energy increases, other things being equal. This may exclude the applicability of the DR deflector method

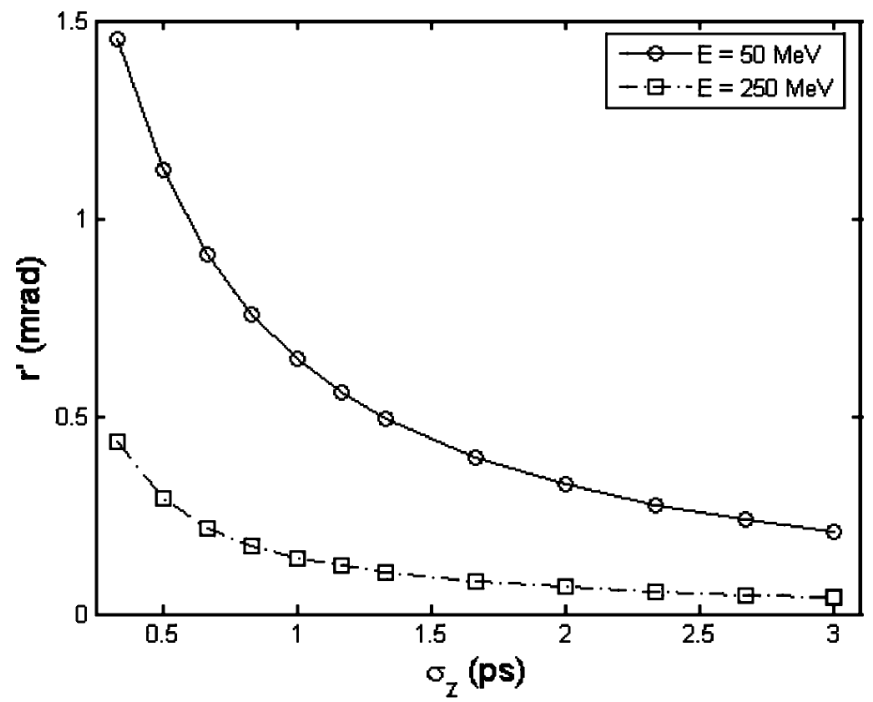

FIG. 2. DR Deflection of medium-energy electron beam with various bunch length. from high-energy electron bunch length measurement. However, when the bunch length is sufficiently short, the deflection could be large enough to be easily detected even for a high-energy beam. Take the LCLS BC2, for example, the beam energy is $4.54 \mathrm{GeV}$, the bunch length before and after the BC2 is about 600 and $70 \mathrm{fs}$, respectively [6]. The deflection from a DR radiator with inner radius $1 \mathrm{~mm}$ and outer radius $30 \mathrm{~mm}$ is shown in Fig. 3 for various bunch length. The deflection is still observable with a typical BPM and the bunch length could also be inferred.

Another important application of short electron bunch is UED which aims at direct observation of fundamental structural transitions that is one of the ultimate goals in scientific fields including nanoscience, chemistry, and biology. To break through the ps barrier and achieve fs temporal resolution, the use of a low charge beam $(<10 \mathrm{pC})$ with a few $\mathrm{MeV}(\sim 2-5 \mathrm{MeV})$ energy and short temporal duration $(\sim 100 \mathrm{fs})$ seems a must [1-3].

Measurement of the bunch length for such low charge low energy and short beam also greatly challenges the beam diagnostic method. The existed methods may fail due to the limited temporal resolution, the weak signal or the combination of them. For instance, the $100 \mathrm{fs}$ temporal duration is beyond the resolution of streak camera and electro-optic sampling method. The measurement of coherent radiation with interferometer or spectrometer is suffered by the weak signal because of two effects. First, the power of coherent radiation is proportional to the square of the charge, so when the charge is decreased by $n$ times the radiation power reduces by $n^{2}$ times. Second, not all of the radiation could be extracted and reach the detector. Instead, the signal is collected within some acceptance angle. So when the energy is low, the radiation cone becomes large and the fraction that reaches the detector is smaller compared to the case for high energy.

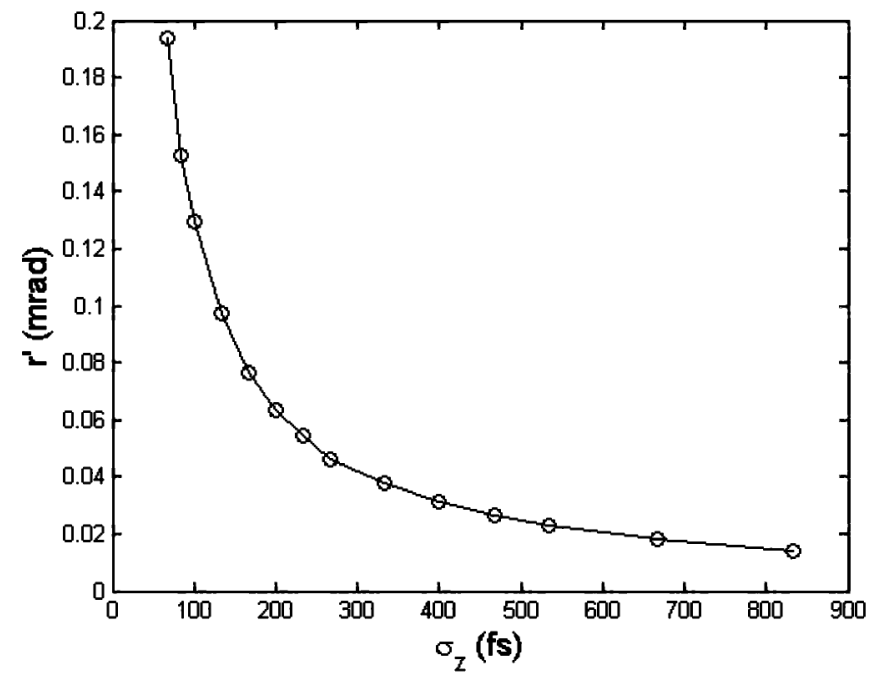

FIG. 3. DR Deflection of high-energy beam with various bunch length. 
These problems are to some extent smoothed in the DR deflector scheme. Even though the radiation power decreases dramatically when beam charge is reduced, taking into account the fact that the electron number is divided in calculation of the deflection as shown in Eq. (2), we get a conclusion that the deflection only decreases linearly as the beam charge is reduced. Furthermore, since the radiation in the whole space all affects the deflection, the fact that the radiation cone is larger for lower energy case may only play a very small role. For instance, we consider the typical parameters in the proposed UED with MeV beam. The beam energy is assumed to be $5 \mathrm{MeV}$, beam charge of $10 \mathrm{pC}$, and bunch length $100 \mathrm{fs}$. When the electron beam passes through the DR radiator with inner radius $0.5 \mathrm{~mm}$ and outer radius $30 \mathrm{~mm}$, the deflection is found to be as large as $0.13 \mathrm{mrad}$, which is easily observable even with the common BPM.

\section{PRACTICAL CONSIDERATIONS}

In order to make the DR deflector practically applicable in bunch length measurement, several relevant issues require investigation. In the section above, the energy spread of the beam is neglected and the beam energy is assumed to be known accurately. We have also assumed the beam with no transverse size. However, for real cases the beams always have energy spread and the energy may change due to fluctuations of RF power and the launching phase of the photoelectron with respect to the RF field. Also the transverse size should be properly considered, for some times it could be larger than the longitudinal extension. In addition to the DR radiator, the trajectory variation could be caused by other factors. In this section we will estimate the practicality of this method with those issues taken into account.

To get a quantitative estimation of the sensitivity of transverse deflection on beam energy, the deflection is calculated for three sets of parameters: $1 \mathrm{nC}$ charge with 1 ps rms bunch length, $500 \mathrm{pC}$ charge with $500 \mathrm{fs}$ bunch length, and $100 \mathrm{pC}$ charge with $200 \mathrm{fs}$ bunch length. The parameters of the DR radiator are the same as those used in Fig. 1 and the results are shown in Fig. 4. The weak dependence on beam energy indicates that the error caused by finite energy fluctuations and energy spread may be neglected for standard modern accelerators.

The transverse beam size will influence the form factor and generally results in a smaller deflection as compared to the pencil beam case. To address this issue, we need to use the 3-dimensional form factor [25],

$$
\begin{aligned}
F\left(\omega, \sigma_{r}, \sigma_{z}, \theta\right)= & \exp \left(-\omega^{2} \sigma_{r}^{2} \sin ^{2} \theta / c^{2}\right) \\
& \times \exp \left(-\omega^{2} \sigma_{z}{ }^{2} \cos ^{2} \theta / c^{2}\right),
\end{aligned}
$$

where we have assumed Gaussian distribution with rms beam size $\sigma_{r}$ for the cross section of the beam.

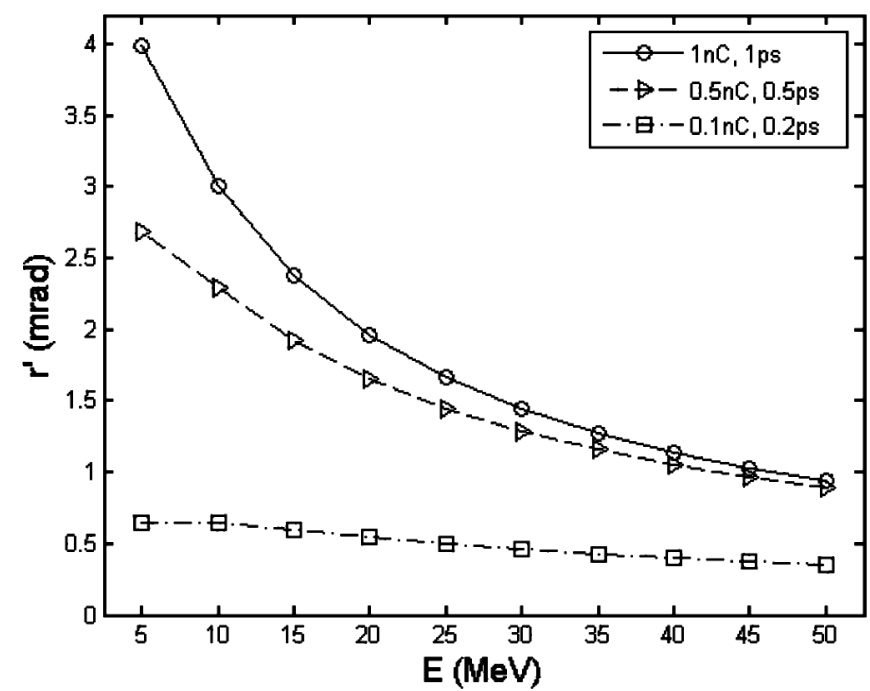

FIG. 4. The dependence of deflection on beam energy with other things being equal.

Equation (3) suggests that, when the condition $4 \pi^{2} \sin ^{2} \theta \sigma_{r}^{2} / \lambda^{2} \ll 1$ is satisfied, the transverse beam size effect could be neglected. The coherence starts at about $\lambda \approx 2 \sigma_{z}$, so we may say if most of the kick is from the photons for which the polar angle is smaller than $\theta_{\text {th }}$, the beam cross section effect could be neglected. The threshold angle is approximately found to be

$$
\theta_{\mathrm{th}} \approx 0.1 \sigma_{z} / \sigma_{r}
$$

Equation (4) indicates that the transverse beam size effect is more significant for beam with a smaller $\sigma_{z} / \sigma_{r}$ ratio. That is to say, for a beam with a short bunch length and a large transverse cross section, the transverse beam size effect should be properly considered. Substituting Eq. (3) into Eq. (2), we can quantitatively estimate the

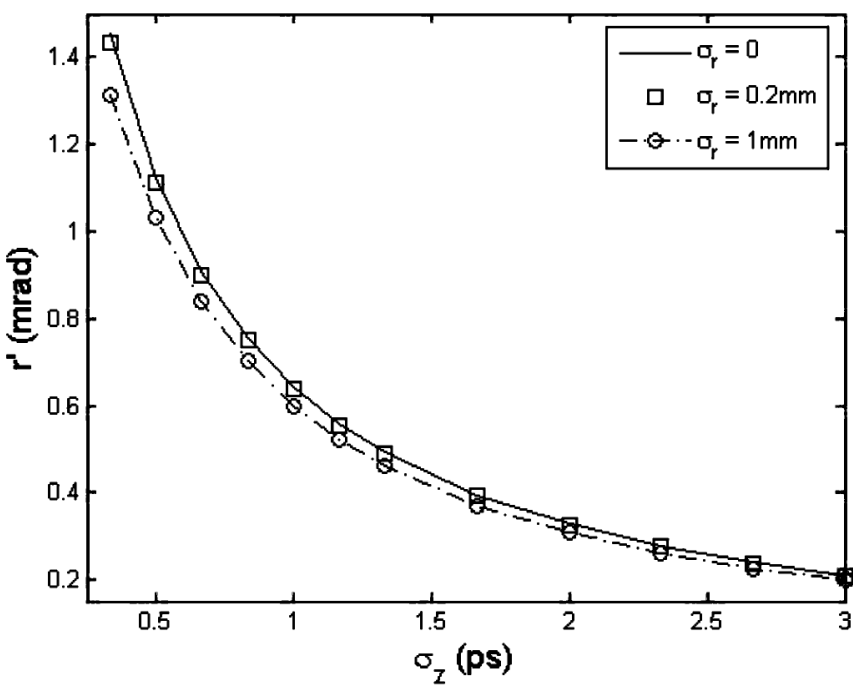

FIG. 5. Influence of transverse beam size on DR deflection. 
DR deflection for beams with various transverse sizes. The results for the $50 \mathrm{MeV}$ and $1 \mathrm{nC}$ case are shown in Fig. 5.

From Fig. 5, we could see, even for the beam with $1 \mathrm{~mm}$ transverse size, there is only a tiny difference from that of a pencil beam. For standard modern accelerators, to make beam size smaller than $0.2 \mathrm{~mm}$ is regularly achievable and thus the transverse beam size should not bother the bunch length measurement with DR deflector.

During the measurement, the BPM downstream also records the trajectory variation caused by other factors upstream of the DR deflector, e.g., laser position jitter for a photocathode RF gun, etc. So we suggest using another 2 BPMs upstream of the DR deflector to record trajectory variations due to other factors. By comparing the results of the BPMs, we should be allowed to extract the deflection caused by the DR deflector alone and an accurate measurement of bunch length and real-time monitoring the bunch length variation could be achieved.

\section{SUMMARY AND CONCLUSIONS}

In this paper, we proposed a new DR based method for electron bunch length measurement. The method uses a DR radiator to deflect the beam and a downstream BPM to measure the deflection. It is found that the deflection is very sensitive to the electron bunch length. Detailed investigations show that this method has wide applicability, high temporal resolution, and great simplicity.

\section{ACKNOWLEDGMENTS}

The authors would like to thank X.J. Wang, T. Watanabe, and J. Y. Huang for helpful discussions and comments. This work was supported by Chinese National Foundation of Natural Sciences under Contract No. 10475047.
[1] Hyotcherl Ihee et al., Science 291, 458 (2001).

[2] X. J. Wang, D. Xiang, T. K. Kim, and H. Ihee, J. Korean Phys. Soc. 48, 390 (2006).

[3] J. B. Hastings et al., Appl. Phys. Lett. 89, 184109 (2006).

[4] R. W. Schoenlein et al., Science 274, 236 (1996).

[5] M. J. van der Wiel et al., Phil. Trans. R. Soc. A 364, 679 (2006).

[6] LCLS Conceptual Design Report, SLAC-R-593, 2002.

[7] Mitsuru Uesaka, Toru Ueda, Takahiro Kozawa, and Toshiaki Kobayashi, Nucl. Instrum. Methods Phys. Res., Sect. A 406, 371 (1998).

[8] D. X. Wang, G. A. Krafft, and C. K. Cinclair, Phys. Rev. E 57, 2283 (1998).

[9] X. Yan et al., Phys. Rev. Lett. 85, 3404 (2000).

[10] I. Wilke et al., Phys. Rev. Lett. 88, 124801 (2002).

[11] G. Berden et al., Phys. Rev. Lett. 93, 114802 (2004).

[12] A. L. Cavalieri et al., Phys. Rev. Lett. 94, 114801 (2005).

[13] Sara Casalbuoni et al., TESLA Report 2005-01.

[14] R. Lai and A. J. Sievers, Phys. Rev. E 50, R4294 (1994).

[15] Hung-Chi Lihn et al., Phys. Rev. E 53, 6413 (1996).

[16] M. Castellano et al., Phys. Rev. E 63, 056501 (2001).

[17] A. H. Lumpkin, N.S. Sereno, and D.W. Rule, Nucl. Instrum. Methods Phys. Res., Sect. A 475, 470 (2001).

[18] Yu. N. Dnestrovskii and D. P. Kostomarov, Dokl. Akad. Nauk SSSR 124, 792 (1959).

[19] Dao Xiang and Wen-Hui Huang, Nucl. Instrum. Methods Phys. Res., Sect. B 248, 163 (2006).

[20] Dao Xiang and Wen-Hui Huang, Nucl. Instrum. Methods Phys. Res., Sect. A 553, 381 (2005).

[21] Dao Xiang et al. (unpublished).

[22] A. Potylitsyn et al., Nucl. Instrum. Methods Phys. Res., Sect. B 227, 170 (2005).

[23] Dao Xiang and Wen-Hui Huang, Nucl. Instrum. Methods Phys. Res., Sect. B 240, 855 (2005).

[24] V. Balakin et al., Proceedings of the 1999 Particle Accelerator Conference, New York, 1999, p. 461.

[25] Hung-Chi Lihn, Ph.D. dissertation, Stanford University [SLAC-R-480, 1996 (unpublished)]. 